\title{
Recognition memory for related pictures
}

\author{
KATHY PEZDEK \\ California State College, San Bernardino, California 92407
}

\begin{abstract}
This study presents a test of the notion of semantic integration of information in pictures. In Experiment 1, subjects were presented pictures which comprised various subsets of the elements in one of four standard pictures. In the test phase, recognition confidence for old and new items was a direct function of the number of figures in the test picture. However, recognition confidence was significantly higher for old than for new pictures, contrary to predictions of the integration model of Bransford and Franks (1971). In Experiment 2, the differential predictions of the integration model and the tally model (Reitman \& Bower, 1973) were tested. The information for a key standard picture was presented solely as ONEs during acquisition. For the other three standard pictures, information was presented as ONEs, TWOs, and THREEs. The results were that recognition confidence increased with complexity of the test item with regular items but decreased with increasing complexity with key items. Together, these experiments support the tally model and demonstrate the generalizability of this model to handle pictorial materials. Implications regarding the nature of picture memory are also discussed.
\end{abstract}

Until recently, the notion of semantic integration of information in memory has been largely associated with processing verbal materials (e.g., Barclay, 1973; Bransford \& Franks, 1971; Honeck, 1973; and with children, Barclay \& Reid, 1974). The results of these studies suggest that subjects spontaneously synthesize the information expressed in individual nonconsecutive sentences into holistic mental representations which transcend the meaning expressed in the individual sentences.

The Bransford and Franks (1971) study demonstrates the principle of semantic integration and, because of its relevance to the present experiment, will be described here. In the acquisition phase of Experiment 2 in their study, subjects were presented sentences which encompassed various subsets of the information expressed in one of a number of complex sentences. One such complex sentence is, The tall tree in the front yard shaded the man who was smoking his pipe. Subjects were not presented this complex sentence during acquisition, but saw only sentences that expressed one, two, or three of the possible four relationships in the complex sentence. In a later recognition test, subjects saw old and new sentences that were constructed as described above. In addition, in the test phase, the whole complex sentences were presented for the first time; five NONCASE sentences were also

This research was supported by Spencer Foundation Grant B-374 to the author. I would like to thank Darrell Butler for his help in collecting the data for Experiment 2 and Leah Light and Ovid Tzeng for their thoughtful suggestions. I am also grateful to Mary Newman for her creative contribution of the pictorial materials. Requests for reprints should be sent to Kathy Pezdek, Department of Psychology, California State College, San Bernardino, California 92407. presented. The NONCASEs were constructed by combining information from across complex sentences. The results were that subjects' confidence in classifying test sentences as old was found to be a function of the number of relationships expressed in the test sentence, and old sentences were not recognized as old more confidently than were new sentences. The more similar the test sentence was to the corresponding complex sentence, the more confident were subjects that they had seen the sentence in the presentation phase. The results of Bransford and Franks are presented in Figure 1. The data here have been collapsed from the relevant conditions in Experiments 2 and 3 of their

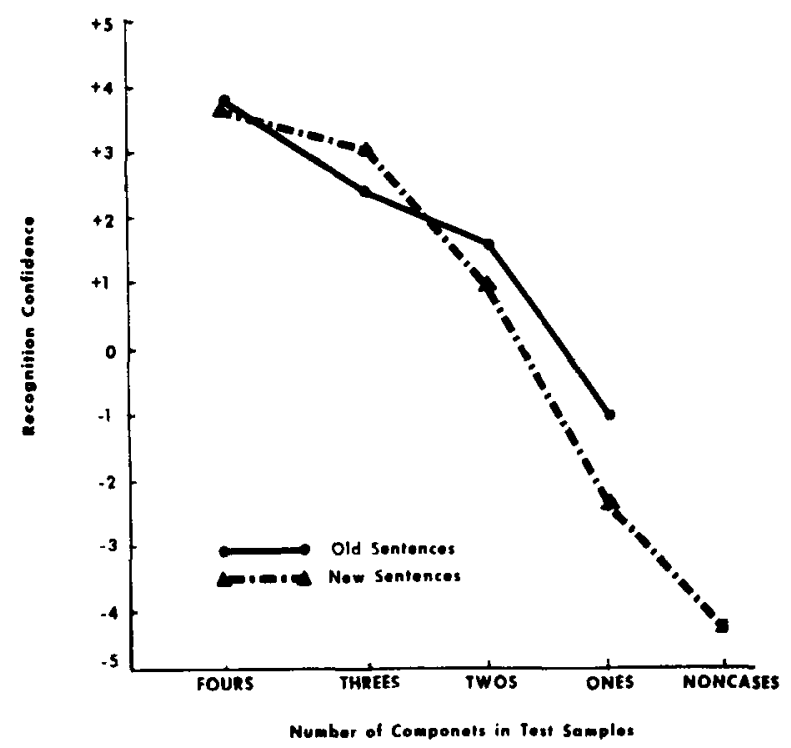

Figure 1. Recognition confidence as a function of the number of components in the test sentences, reported by Bransford and Franks (1971). 
study. The explanation of these results was that subjects spontaneously integrated the individual sentences in memory and made subsequent recognition decisions on the basis of how well each test sentence matched the holistic representation.

The present study is aimed at determining the extent to which the notion of semantic integration is applicable to memory for pictorial materials. A convincing body of research exists which demonstrates subjects' impressive ability to recognize (e.g., Nickerson, 1965; Shepard, 1967; Standing, Conezio, \& Haber, 1970) and recall (Bousfield, Esterson, \& Whitmarsh, 1957) pictorial materials. This research might suggest that, if subjects do synthesize information from related pictures, they also retain very good visual memory for the original pictures presented. However, the pictorial materials in these studies were individual unrelated pictures. The present study tests the generalizability of this result to a situation in which pictorial sequences consist of pictures that are visually and semantically related to each other and include partially redundant information.

Recently, evidence has emerged supporting subjects' ability to synthesize aspects of visual forms in memory (Franks \& Bransford, 1971; Palmer, 1975), integrate features of multifeature visual stimuli (Klatzky \& Thompson, 1975), and integrate semantically relevant visual and verbal materials (Pezdek, 1977). Together this research suggests that, although subjects' memory for individual pictures is usually very good, when the stimulus sequence includes related pictures or very similar physical forms, or when the task demands such, subjects can integrate aspects of visual stimuli. The present study is concerned with whether subjects spontaneously integrate aspects of drawings of realworld scenes in a recognition task in which they have been instructed to try to remember each picture.

Experiment 1 was designed to be comparable to the Bransford and Franks (1971) study, but instead used pictorial materials. In the presentation phase, subjects saw pictures which were constructed by combining various subsets of the information from one of the four standard pictures; however, the standard pictures, which included the complex pictures from which all subsets were derived, were not presented. In the recognition test that followed, subjects were presented pictures, half of which had been presented in the first part of the experiment and half of which were new. The set of new pictures was constructed similarly to the old pictures, with the inclusion of the four standard pictures and NONCASEs.

An important procedural difference between this experiment and that by Bransford and Franks is that in the latter study, as each sentence was presented, subjects (1) listened to the sentence, (2) performed a color-naming task, and (3) answered a question concerning the sentence just read. Subjects were not told that they were to try to remember the sentences for a later recognition test. To create a test of spontaneous inte- gration in the present study, subjects were instructed to concentrate on remembering each picture as it was presented.

If subjects spontaneously integrate the corresponding pictures in the presentation phase, then what subjects retain in memory could be described as similar to the standard picture for each set. In the subsequent recognition test, then, subjects should (1) be unable to differentiate old and new items and (2) be more confident in recognizing pictures that approximate the standard picture rather than the original pictures presented. Such an outcome would produce results similar to those of Bransford and Franks (1971) as presented in Figure 1. If subjects' recognition memory is simply a function of how well test items compare with their memory of the presentation items, then the predicted result would be no change in recognition confidence as a function of the number of relations expressed in test pictures.

\section{EXPERIMENT 1}

\section{Method}

Subjects. The subjects were 20 undergraduates enrolled in psychology courses at California State College, San Bernardino. They were tested in groups of three to five subjects each. The 20 subjects were arbitrarily divided, with each half receiving a different order of presentation of the test slides. All other variables were manipulated as within-subjects variables. At the time of selection, subjects were told only that they would be participating in an experiment in memory for pictures.

Materials. Four standard pictures were constructed, each having five interacting figures on a simple contextual background. The pictures were drawn in black on a white background. The content of the four standard pictures was highly dissimilar to avoid confusion of figures across sets. An example of one of the standard pictures and a sample of exemplars are presented in Figure 2. From each of the standard pictures, 11 exemplars were drawn. Each exemplar was identical to the corresponding standard except that it included a subset of items in the original figure, expressing the relationship between two (referred to as the ONEs), three (TWOs), or four (THREEs)
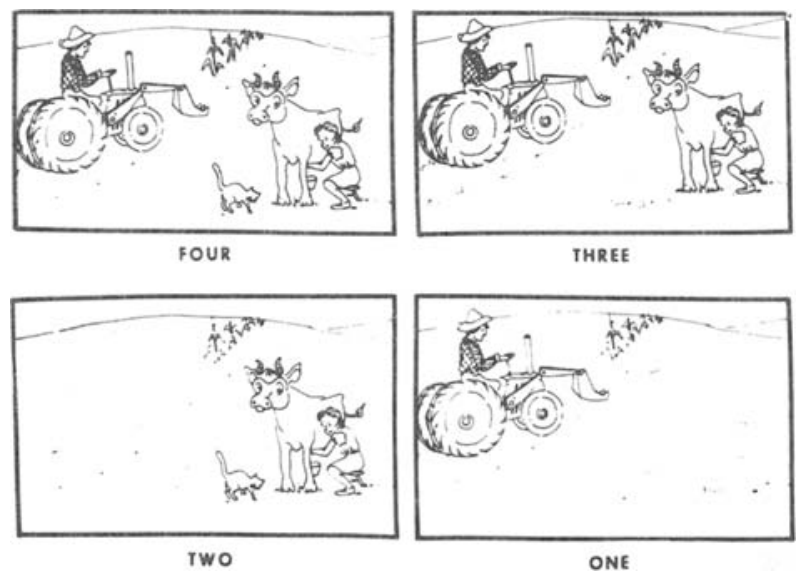

Figure 2. Example of a standard complex picture (FOUR) and corresponding exemplars. 
of the original five figures which comprised the whole picture. In each of the exemplars, the figures included in the picture were positioned on the contextual background exactly as in the whole picture, with the only difference being which figures were deleted. In each subset of ONEs, TWOs, and THREEs, pictures were constructed in which the specified number of figures was randomly selected from the total of five possible figures. For each standard picture there were constructed four ONEs, four TWOs, three THREEs, and one FOUR (the holistic standard). In addition, there were four NONCASEs. Each NONCASE was a picture constructed by combining five figures from more than one of the standard pictures while maintaining each figure in its original position in the slide.

Procedure. The experiment consisted of an acquisition phase followed by a delay task and then a test phase. The delay task was a 5-min Peterson and Peterson (1959) counting task in which subjects counted backward by threes from randomly designated numbers and wrote the number sequences on a sheet of paper.

In the acquisition phase, subjects saw 24 pictures which were exposed for $3 \mathrm{sec}$ each, with $5 \mathrm{sec}$ between slides with the shutter closed. This sequence was regulated by a preprogrammed millisecond timer attached to the shutter of a Kodak Carousel slide projector. The pictures in the acquisition phase were two ONEs, two TWOs, and two THREEs selected from each of the four standard sets. The order arrangement of the 24 pictures was randomly blocked such that, within each block of four, one randomly selected slide was included from each of the four basic sets. All subjects were presented the slides in the acquisition phase in the same order. Subjects were instructed to concentrate on remembering each picture as it was presented.

In the test phase, subjects were presented 48 slides, twice each, for a total of 96 test pictures in a continuous sequence. The first 48 test slides are referred to as Trial 1, the second 48 as Trial 2 . The test pictures included the 24 pictures from the acquisition phase plus 24 new pictures. The new pictures were two ONEs, one TWO, one THREE, one FOUR and one NONCASE from each of the four standard sets. The order of the test slides was block randomized as in the presentation phase. For Group 1 the order of the test slides was 148 , 48-1. For Group 2 the order was 1-48, 1-48.

In the test phase, slides were presented for $4 \mathrm{sec}$, with $5 \mathrm{sec}$ between slides with the shutter closed. During this time subjects responded on their protocol sheets as to whether each picture was "old," that is, one that they had seen in the first part of the experiment, or "new." In addition, subjects were asked to rate their confidence in making each response on a scale from one (very high) to five (very low).

\section{Results}

Recognition confidence ratings were converted into numerical values for the purpose of analysis. Subjects' responses were transformed into a 1 to 10 scale, where 1 was "yes" with highest confidence, 10 was "no" with highest confidence, and the values in between represented responses of increasingly less certainty. An average was then calculated for items within each condition for each subject. The data, transformed in this way and averaged over subjects, are presented in Figure 3.

Three analyses of variance were applied to the data. The first assessed responses to new test items only. The second assessed responses to old test items. The third analyzed responses to old and new items together on the ONEs, TWOs, and THREEs. In each

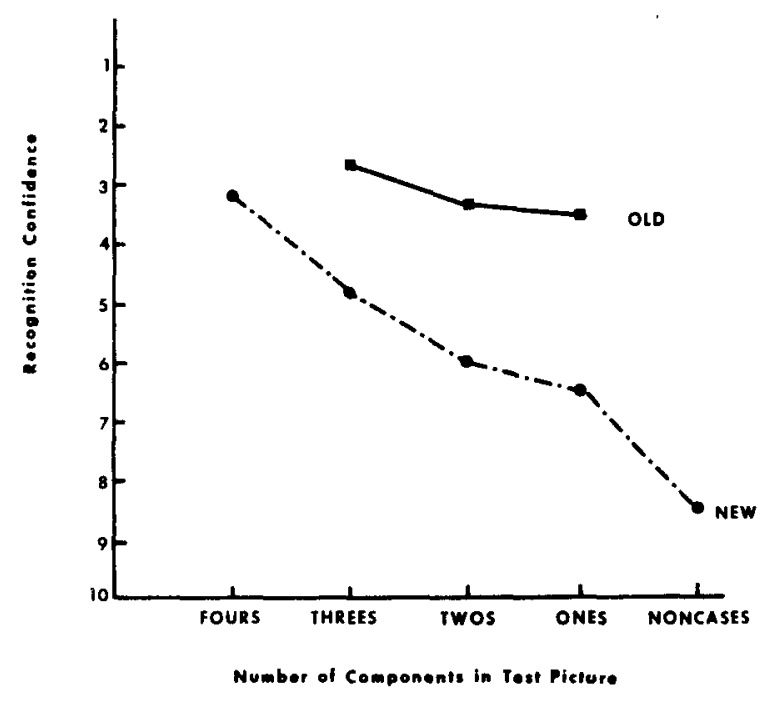

Figure 3. Recognition confidence ratings for old and new test pictures, Experiment 1.

analysis the factors included were order of presentation (Group 1 or Group 2), trial number (1 and 2), and number of components in the test stimulus. All comparisons reported were significant at the .05 level or better.

In the analysis of the new items, the result of primary interest was the effect of the number of components in the picture on recognition confidence. As can be seen in Figure 3, the closer the new test picture approximated the complex picture, the more confident were subjects that they had seen the picture before. This finding was highly significant $[F(4,72)=$ $62.96, \mathrm{MSe}=2.53 \mathrm{l}$. The absence of an effect of trial number on recognition confidence suggests that the effect of number of components was consistent throughout testing.

The analysis of old test items also resulted in a significant main effect of number of components in the test picture $[\mathrm{F}(2,36)=5.60, \mathrm{MSe}=.97]$. This effect was in the predicted direction, as can be seen in Figure 3, with subjects most confident in recognizing THREEs, then TWOs, and least confident in recognizing ONEs. The only other significant effect was that subjects' overall recognition confidence decreased from Trial 1 (mean response $=2.77$ ) to Trial $2(3.28)[\mathrm{F}(1,18)=12.02, \mathrm{MSe}=.66]$.

The analysis of the combined old and new items was performed on the ONEs, TWOs, and THREEs, as the FOURs and NONCASEs were included as new test items only. In this analysis the effect of the number of components in the test picture was significant $[\mathrm{F}(2,36)=27.50$, MSe $=1.06]$. As can be seen in Figure 3, the difference between subjects' recognition confidence with old (3.03) vs. new (5.71) pictures was also highly significant $[F(1,18)=273.80$, $\mathrm{MSe}=1.58]$. In terms of percent correct measures, subjects were responding with $79.9 \%$ accuracy with 
old items as compared with $55.7 \%$ accuracy with new items. The interaction of the number of components in the test picture by old/new items was significant $[\mathrm{F}(2,36)=4.18, \mathrm{MSe}=1.08]$ in the direction that the effect of the number of components in the picture on recognition confidence was greater with new than with old test items. The only other significant effect in this analysis was the main effect of trials $[F(1,18)=$ $8.23, \mathrm{MSe}=1.28]$, with recognition confidence less on Trial 2 than on Trial 1.

A final one-way analysis of variance was carried out to test if subjects responded differently to subsets of the four different complex pictures utilized. This contrast was not significant, suggesting that the results were generalizable across the different pictures.

\section{Discussion}

The purpose of the present study was to determine if subjects spontaneously integrate semantic aspects of pictures in memory. If subjects integrated the information presented in the corresponding pictures in the presentation phase, what they would have in memory would be similar to the complex picture for each set. In the recognition test, then, subjects would respond on the basis of how well each test picture matched the complex picture in memory. As a result, it would be predicted that recognition confidence would increase with the number of components in the test item, and that subjects would not differentiate old and new items, as was the case in the Bransford and Franks (1971) results shown in Figure 1.

The essential results of Experimentl are presented in Figure 3. In responding to new test items, subjects performed almost identically to the subjects in the Bransford and Franks study. Although this result alone might lend support to the notion of semantic integration, the results on old test items raise considerable doubt. Old items were recognized significantly more accurately ( $79.9 \%$ correct) than were new items $(55.7 \%)$. The high rate of response accuracy with old items indicates that, to some extent, subjects remembered the items presented during acquisition.

Bransford and Franks have argued that, in the process of integrating relevant materials, subjects do not retain information about the acquisition items. As a result, no difference in recognition confidence to old and new test items would be predicted by the integration model. Although this result was obtained by Bransford and Franks, numerous studies have recently reported higher recognition confidence for old than new items using meaningful verbal materials (Flagg, 1976; Flagg \& Reynolds, 1977; Katz \& Gruenewald, 1974) and alphanumeric strings (Flagg, 1976; Reitman \& Bower, 1973). The old-new split in the results of Experiment 1 are consistent with these latter findings and question the adequacy of the integration interpretation of the data.
An alternative explanation of the monotonic function obtained by Bransford and Franks, and the above mentioned studies, has been proposed by Reitman and Bower (1973). This model assumes that as each item is presented subjects store the whole item in memory but that the tag "generalizes" to the n-grams composing the item. Using their example, if the item 123 was presented, strength tags associated in memory with the n-grams $1,2,3,12,23$, and 123 would be incremented. At the time of test, subjects would search memory for the individual components of the test item, checking with repeated passes first the component monograms, then digrams, then trigrams, etc. With each pass, the strength tag associated with each $\mathrm{n}$-gram would be tallied. The tally would be incremented if the strength tag associated with an n-gram was above some threshold; the tally would otherwise be decremented. When all derivable n-grams of a test item had been scanned, if the resulting tally was above some cutoff, a response of "old" would be made. Test items with more components would be responded to with a higher recognition confidence because, although the items were not seen before in totality, they contained more n-grams, many of which were presented during acquisition.

Flagg (1976) conducted a crucial test of the adequacy of an integrative, as compared with a tally, model to account for the monotonic effect observed by Bransford and Franks and subsequent studies. When all of the information about a complex idea is presented solely as ONEs during acquisition, the two models make different predictions. The tally model predicts that since the memory representation for this standard idea consists only of monograms, the probability of an "old" response would not be increased by the additional higher order n-grams derivable from the test item. The tally, in fact, would be decremented with passes to any higher order n-grams. As a result, subjects would respond with a high recognition confidence to test items which were ONEs and with decreasing recognition confidence to test items with progressively more components.

Bransford and Franks' model would predict that the ONEs presented during acquisition would be integrated in memory because of their semantic relatedness. At the time of test, then, the model would predict increased recognition confidence with an increased number of components in the test item.

The results obtained by Flagg (1976) using both sentences and alphanumeric strings were consistent with the predictions of the tally model. With the set of items originally presented solely as ONEs, at the time of test subjects responded with high recognition confidence to ONEs and with significantly decreasing recognition confidence to test items which were TWOs, THREEs, and FOUR. Experiment 2 was conducted to test the tally model as an alternative explanation 
of the results of Experiment 1. The procedure utilized largely replicated that of Flagg, with the obvious change that pictures were used as the stimulus materials. The notion that pictures might be stored in memory in such a way that they could be scanned and tallied would provide an interesting addition to the literature on picture memory.

\section{EXPERIMENT 2}

\section{Method}

Subjects and Design. The subjects were 31 students recruited from classes at California State College, San Bernardino. They were tested in groups of three to six subjects each. The order of presenting the acquisition items was block-randomly arranged in four orders. Eight subjects received each order of presentation. Because of failure to follow instructions, one subject was eliminated from the experiment, resulting in only seven subjects in one group. All other variables were manipulated as within-subjects variables.

Materials. The four standard pictures from Experiment 1 provided the basis for the material used in this experiment. One of the four standard pictures was indicated as the "key" standard and the other three were the "regular" standards. The specific standard picture which served as the key standard was counterbalanced across the four orders of presentation. For the key standard, all of the information pertaining to that picture was presented solely as ONEs during acquisition. From the key standard, subjects were presented four ONEs, twice each. For each of the three regular standard pictures, subjects were presented one ONE, two TWOs, and one THREE. The acquisition list thus consisted of 20 pictures. The pictures derived from each regular standard were selected such that each of the five figures in the standard was presented twice.

The test sentences for each standard picture consisted of four ONEs, two TWOs, two THREEs, and the FOUR. For the key standard, the four ONEs were old items and all other test items were new. For the regular standard, a single ONE, TWO, and THREE were old and the remaining test items were new. The test sequence also included five NONCASEs, for a total of 41 pictures. The order of presenting the test items was block randomized, with the NONCASEs randomly inserted in the sequence. All subjects received the test items in the same order. In both the acquisition and test sequences, no two consecutive items were from the same standard picture.

Procedure. The experiment consisted of an acquisition phase followed by a delay task and then a test phase. The delay task was a 10-min maze-tracing task. The stimulus materials were on slides projected on a screen by a Kodak Carousel slide projector. The 20 acquisition slides were exposed for $4 \mathrm{sec}$ each, with $3 \mathrm{sec}$ between slides with the shutter closed. Subjects were instructed to concentrate on remembering each picture as it was presented.

In the test phase, slides were exposed for $7 \mathrm{sec}$, with $2 \mathrm{sec}$ between slides. During this time subjects responded on their protocol sheets as to whether each picture was old or new; additionally, subjects rated their confidence in making each response on a 5-point rating scale.

\section{Results}

The recognition confidence ratings were converted to numerical values as in Experiment 1. The data, averaged over all subjects, are presented in Figure 4 .

The pattern of results obtained with regular test items was similar to the results of Experiment 1. NONCASEs were detected very accurately $\mathbf{( 9 6 . 7 8 \%}$

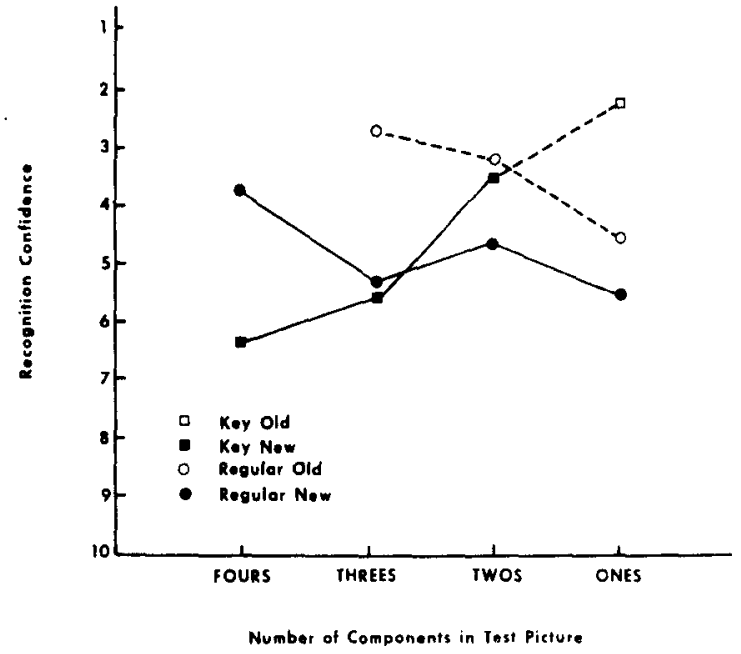

Figure 4. Recognition confidence ratings for regular and key pictures, Experiment 2.

correct). Old regular items were recognized more accurately $(73.48 \%$ correct) than new regular items (48.03\%). Also, recognition confidence increased with increased complexity of the test item. An analysis of variance was carried out on the recognition confidence data for old and new regular items at complexity levels ONE, TWO, and THREE. The effect of old vs. new items was significant $[\mathrm{F}(1,30)=57.78, \mathrm{MSe}=2.28]$, as was the effect of complexity $[F(2,60)=9.98$, $\mathrm{MSe}=2.55]$. The interaction of complexity by old $/$ new item was also significant $[\mathrm{F}(2,60)=3.44$, MSe $=3.08]$. The interaction appeared to be due to the relatively low recognition confidence for the new regular pictures with THREE components. There is no obvious explanation for the deviation in monotonicity of this point.

The critical manipulation in Experiment 2 is examined in subjects' responses to the key test items. The differences in recognition confidence for key items across levels ONE through FOUR were highly significant $[\mathrm{F}(3,90)=24.46, \mathrm{MSe}=4.56]$. Recognition was highest for the ONEs (all of which were old) and decreased as the complexity of the new key items increased. Recognition confidence on new key items alone also decreased significantly from TWO through FOUR $[F(2,60)=22.22, \mathrm{MSe}=3.36]$. As can be seen in Figure 4, the slope of the line indicating subjects' recognition confidence to key items was in the opposite direction from the responses to old and new regular items. This reversal in the slope for key items is inconsistent with the predictions of the integration model.

\section{GENERAL DISCUSSION}

The results of Experiment 1 and with regular items in Experiment 2 are generally compatible with the findings of Flagg (1976), Katz and Gruenewald (1974), and Reitman and Bower (1973). Recognition confidence increased with complexity, and there was a split in 
recognition confidence between old and new test items. This suggests that the pictorial stimuli in the present study were processed similarly to the sentences and alphanumeric strings used in the other studies, rather than each picture being preserved in memory holistically in the form in which it was presented.

The discrepancy in recognition confidence between old and new test pictures in Experiment 1 and with the regular items in Experiment 2 raised doubt as to the adequacy of the integration model to account for the data. The alternative tally model proposed by Reitman and Bower (1973) was tested by including the key items in Experiment 2, as suggested by Flagg (1976). The result, that recognition confidence to key pictures decreased as complexity increased, supports the tally model and not the integration model.

The interpretation of the reversed monotonic function for key items follows. The drop in recognition confidence from ONEs to TWOs could be accounted for by the general old-new split. However, the integration position would then predict that recognition confidence would increase from TWO to FOUR. The tally model, on the other hand, would predict that recognition confidence would decrease as complexity increased from TWO to FOUR. This latter prediction describes the obtained result.

Application of the tally model to the results of the present study has some interesting implications regarding the nature of picture memory. Previous studies on picture memory (cf. Nickerson, 1965) have used long series of individual, unrelated pictures to demonstrate subjects' highly accurate and enduring memory for pictures. The assumption of these earlier studies was that the visual features of pictures are retained in memory, and it is because of the uniqueness of these visual features within each picture that the memory is so good. The sequence of pictures in the present study included pictures that were related to each other, and in some cases the pictures even presented partially redundant information. Under these conditions, picture memory was not found to be superior to memory for sentences reported by Bransford and Franks (1971) and Flagg (1976) using a similar task. Further, the results of the present study suggest that the pictures were not stored in memory in such a way that the visual features of each picture were uniquely retained. The components of each picture appear to have been stored and scanned in a manner consistent with the tally model. The results of earlier studies demonstrating excellent long-term memory for pictures may be restricted to a limited type of pictorial material.

In summary, the monotonic relationship between complexity and recognition confidence is a robust effect which holds up for meaningful pictures as well as for other types of stimulus materials. The integration explanation offered by Bransford and Franks, however, does not adequately explain the results obtained. Semantic integration across pictures was not supported. The tally model presented by Reitman and Bower, and more general models of this type (cf. Anderson \& Bower, 1973, pp. 345-352), explain the data handily and, at this point, provide the most convincing interpretation of the results.

\section{REFERENCES}

Anderson, J. R., \& Bower. G. H. Human associative memory. Washington, D.C: V. H. Winston, 1973.

BARCLAY. J. R. The role of comprehension in remembering sentences. Cognitive Psychology, 1973, 4. 229-254.

Barclay, J. R., \& REID, M. Characteristics of memory representations of sentence sets describing linear arrays. Journal of Verbal Learning and Verbal Behavior, 1974, 13, 133-137.

Bousfield, W. A., Esterson, J., \& Whitmarsh, G. A. The effects of concomitant colored and uncolored pictorial representations on the learning of stimulus words. Journal of Applied Psychology, 1957, 41. 165-168.

BransFord, J. D., \& Franks, J. J. The abstraction of linguistic ideas. Cognitive Psychology, 1971, 2, 331-350.

FLAGG, P. W. Semantic integration in sentence memory? Journal of Verbal Learning and Verbal Behavior, 1976. 15, 491-504.

FlaGg. P. W., \& Reynolds. A. G. Modality of presentation and blocking in sentence recognition memory. Memory \& Cognition. 1977. 5, 111-115.

Franks, J. J., \& Bransford, J. D. Abstraction of visual patterns. Journal of Experimental Psychology, 1971, 90, 65-74.

Honeck, R. P. Interpretive versus structural effects on semantic memory. Journal of Verbal Learning and Verbal Behavior, 1973, 12, 428-432.

Katz. S. \& Gruenewald, P. The abstraction of lingusitic ideas in "meaningless" sentences. Memory \& Cognition. 1974, 2. 737.741.

KLATZKY, R. L., \& Thompson, A. Integration of features in comparing multifeature stimuli. Perception \& Psychophysics, 1975, 18, 428-432.

Nickerson. R. S. Short-term memory for complex meaningful visual configurations: $A$ demonstration of capacity. Canadian Journal of Psychology, 1965, 19. $155-160$.

Palmer, S. E. Visual perception and world knowledge: Notes on a model of sensory-cognitive interaction. In D. A. Norman \& D. E. Rumelhart, Explorations in cognition. San Francisco: Freeman, 1975.

Peterson, L. R., \& Peterson, M. J. Short-term retention of individual verbal items. Journal of Experimental Psychology, 1959, 58, 193-198.

PEZDEK, K. Cross-modality semantic integration of sentence and picture memory. Journal of Experimental Psychology: Human Learning and Memory, 1977, 3, 515-524.

Reitman, J. S., \& Bower, G. H. Storage and later recognition of exemplars of concepts. Cognitive Psychology. 1973, 4, 194-206.

SHEPARD, R. N. Recognition memory for words, sentences, and pictures. Journal of Verbal Learning and Verbal Behavior. 1967, 6, 156-163.

Standing, L., Conezio, J., \& Haber. R. N. Perception and memory for pictures: Single-trial learning of 2560 visual stimuli. Psychonomic Science. 1970, 19, 73-74.

(Received for publication May 9, 1977: accepted October 7, 1977.) 